\title{
Superfluid phase diagrams of trapped Fermi gases with population imbalance
}

\author{
Chih-Chun Chien, Qijin Chen, Yan He, and K. Levin \\ James Franck Institute and Department of Physics, University of Chicago, Chicago, Illinois 60637
}

(Dated: August 18, 2018)

\begin{abstract}
We present phase diagrams for population imbalanced, trapped Fermi superfluids near unitarity. In addition to providing quantitative values for the superfluid transition temperature, the pairing onset temperature and the transition line (separating the Sarma and phase separation regimes), we study experimental signatures of these transitions based on density profiles and density differences at the center. Predictions on the BCS side of resonance show unexpected behavior, which should be searched for experimentally.
\end{abstract}

PACS numbers: 03.75.Hh, 03.75.Ss, 74.20.-z

cond-mat/0612103

The study of ultracold trapped Fermi gases, as they vary from BCS to Bose-Einstein condensation (BEC) is a rapidly exploding subject which is defining new directions in condensed matter and atomic physics. With the recent focus on population imbalanced superfluids [1, 2, 3, 4], this body of work has also captured the attention of physicists in other disciplines [5]. Indeed, it is hard in recent years to find a subfield of physics which has such a broad appeal across the different sub-specialties. The goal of the present paper is to address the multiple superfluid and normal phases which appear in these trapped gases and which are viewed as possible prototypes for high temperature superconductors [6], as well as quark and nuclear matter. The various phases we contemplate become stable or unstable as one alters the populations of the two different spin species or changes the temperature $T$. There has been a very extensive theoretical literature on this subject which is almost exclusively confined to $T=0[7,8,8]$. Our emphasis here, is on finite temperature effects [6, 10], which are essential in order to address the actual experimental situation. We do this here along with presenting predictions for new experiments.

The fermionic superfluid ground state wavefunction in the presence of BCS-BEC crossover effects (with population imbalance) is almost universally assumed [7, 8, 9] to be of the BCS-Leggett form. The excitations of this ground state are expected to be particularly interesting in the intermediate or unitary regime where they consist of non-condensed pairs as well as gapped fermions. While these finite momentum pair excitations are frequently omitted in the literature [11, 12], we stress that they play an essential role. Only with their inclusion will one find quantitatively meaningful estimates of $T_{c}$; this temperature is reduced dramatically (relative to a naive mean field estimate) as a result of the opening of an excitation or pairing gap well above the transition. Indeed, the separation of the pairing onset temperature $T^{*}$ and the phase coherence temperature $T_{c}$ is well documented in experiments which are consistent with a "pseudogap" (or normal state excitation gap) in the unitary trapped gases [6].

Without the contribution of non-condensed pairs [6, 13] one often finds bimodal particle distributions at $p=0$. Therefore, theories which ignore these pairs [11, 12] may not be applicable for establishing bimodal distributions associated with population imbalance. Pairing fluctuations were partially included (through the number equation) in Ref. [14] based on the Nozier̀es-Schmitt-Rink (NSR) scheme [15] but in the absence of a trap. However, the finite T NSR treatment is not designed to be consistent with the standard ground state [7, 9, 16]. In the presence of population imbalance, it is now well known that one has to consider homogeneous phases (which we refer to as the "Sarma") and Larkin-OvchinnokovFulde-Ferrell (LOFF) phases [17] as well as phase separation [18]. In addition to these competing phases, the normal phase may appear as a highly correlated or paired state without phase coherence, or as a simple Fermi gas (unpaired) phase.

We begin with the (local) thermodynamical potential (per unit volume) for the allowed states (excluding the more complicated LOFF phase which appears to be of less interest near unitarity and at all but the lowest temperatures [19]). We focus on a two-component Fermi gas in a harmonic trap potential. For a normal or superfluid phase in which pairing correlations are present the thermodynamical potential $\left(\Omega_{t o t}\right)$ consists of two highly inter-dependent contributions: from the gapped fermions $\left(\Omega_{f}\right)$ and the non-condensed pairs or bosons $\left(\Omega_{b}\right)$

$$
\begin{aligned}
& \Omega_{t o t}=\Omega_{f}+\Omega_{b} \\
& \Omega_{f}=-\frac{\Delta^{2}}{U}+\sum_{\mathbf{k}}\left(\xi_{k}-E_{k}\right)-T \sum_{\mathbf{k}, \sigma} \ln \left(1+e^{-E_{k \sigma} / T}\right) \\
& \Omega_{b}=Z \mu_{\text {pair }} \Delta_{p g}^{2}+T \sum_{\mathbf{q}} \ln \left(1-e^{-\tilde{\Omega}_{q} / T}\right) .
\end{aligned}
$$

Competing with this phase is the free Fermi gas phase which has thermodynamical potential $\Omega_{\text {free }}=$ $-T \sum_{\mathbf{k}, \sigma} \ln \left(1+e^{-\xi_{k \sigma} / T}\right)$. The (gapped) fermion and pair dispersions are given respectively by $E_{\mathbf{k}}=\sqrt{\xi_{\mathbf{k}}^{2}+\Delta^{2}}$ for a contact (short-range) pairing interaction with strength $U$ and $\tilde{\Omega}_{q}=q^{2} / 2 M^{*}-\mu_{\text {pair }}$, where $M^{*}$ and $\mu_{\text {pair }}$ are the effective mass and chemical potential of the pairs. Both $M^{*}$ and $Z$ are obtainable from a microscopic $T$-matrix approach[10]. Here $E_{\mathbf{k} \sigma}=E_{\mathbf{k}} \mp h$ and $\xi_{\mathbf{k} \sigma}=\xi_{\mathbf{k}} \mp h$ for spin $\sigma=\uparrow, \downarrow$, respectively, where $\xi_{\mathrm{k}}=k^{2} / 2 m-\mu, k_{B}=\hbar=1$ with $\mu=\left(\mu_{\uparrow}+\mu_{\downarrow}\right) / 2$ and $h=\left(\mu_{\uparrow}-\mu_{\downarrow}\right) / 2$.

We distinguish the order parameter $\Delta_{s c}$ from the total gap $\Delta$. Non-condensed pairs are associated with a pseudogap contribution, $\Delta_{p g}$, to the total gap via $\Delta^{2}=\Delta_{s c}^{2}+\Delta_{p g}^{2}$. The form of $\Omega_{b}$ contains a free boson-like contribution; the pair condensate does not contribute to $\Omega_{b}$ directly. Although it is 
not a necessary assumption, in order to simplify the formal description we assume that $M^{*}$ depends on $T$ only, as is reasonably consistent with our microscopic theory [10] and $\mu_{\text {pair }}$ is a function of $\Delta$ and $T$ only. In the summation over boson momentum q, we will impose a cutoff $q<q_{c}$, where $q_{c}$ is the minimum value of $q$ which satisfies $E_{k}+\xi_{\mathbf{k}+\mathbf{q}}-\tilde{\Omega}_{q}=0$ for at least one value of $\mathbf{k}$; above this momentum, pairs become diffusive by decay into the particle-particle continuum. It should be noted that $\Omega_{f}$ and $\Omega_{b}$ couple only via $\mu_{\text {pair }}$. Above $T_{c}$ this leads to an extra contribution to the gap equation.

Our self-consistent equations can be expressed as variational conditions on $\Omega_{t o t}$, which yield equations for the total excitation gap $\Delta$, the pseudogap $\Delta_{p g}$, the fermion number density $n$ and population imbalance $\delta n$, respectively:

$$
\frac{\partial \Omega_{t o t}}{\partial \Delta}=0 ; \quad \frac{\partial \Omega_{t o t}}{\partial \mu_{\text {pair }}}=0 ; \quad-\frac{\partial \Omega_{t o t}}{\partial \mu}=n ; \quad-\frac{\partial \Omega_{t o t}}{\partial h}=\delta n .
$$

Importantly, below $T_{c}$ we have $\mu_{\text {pair }}=0$, and above $T_{c}$ we have $\Delta=\Delta_{p g}$. These equations reduce to the usual temperature dependent BCS-like equations for the gap and number equations in the literature. The effect of the pseudogap, which is new to the present formalism, has also been extensively discussed in earlier papers [6, 10], although here we have presented it in a different, but equivalent fashion.

In a trap, $T$ and $h$ are independent of the radial position. The distribution of the local $\mu(\mathbf{r})$ is determined by the force balance equation, $-\nabla \bar{p}=n \nabla V_{\text {ext }}$, where $\bar{p}=-\Omega_{\text {tot }}$ is the pressure and $V_{\text {ext }}=\frac{1}{2} m \bar{\omega}^{2} r^{2}$ is the harmonic trap potential with mean angular frequency $\bar{\omega}$. Using the number equation $n=-\partial \Omega_{\text {tot }} / \partial \mu$, we obtain $\nabla \mu(\mathbf{r})=-\nabla V_{\text {ext }}(\mathbf{r})$, or $\mu(\mathbf{r})=\mu_{0}-V_{\text {ext }}(\mathbf{r})$, where $\mu_{0} \equiv \mu(0)$ and $V_{\text {ext }}(0)=0$. This shows that the force balance condition naturally leads to the usual local density approximation (LDA). To ensure meaningful comparisons between states under different conditions, we fix the total particle number $N=\int d^{3} r n(r)$ and the number difference $\delta N$. The Fermi energy $E_{F}=T_{F} \equiv k_{F}^{2} / 2 m$ is defined as that of a non-interacting system with the same $N=N_{\uparrow}+N_{\downarrow}$ at the polarization $p \equiv \delta N / N=0$. We assume $N_{\uparrow}>N_{\downarrow}$ so that $h>0$.

The physical state corresponds to the minimum of $\Omega_{t o t}$ and $\Omega_{\text {free. }}$. At a particular trap radius when (and if) these become equal, the system will have a first order transition from a state in which there is pairing (but not necessarily superfluidity) to an unpaired Fermi gas phase. We assume an infinitely thin interface (i.e., domain wall) for this phase separation. There is as yet no complete microscopic theory for the interface energy, so that we do not include it here. The philosophy behind our treatment of phase separation is very similar to that in previous $T=0$ papers [8, 20] except that we include the important effects of temperature (in a fashion consistent with the well studied BCS-Leggett ground state). Across the interface, we require thermal, chemical, and mechanical equilibrium so that $T, \mu_{\sigma}$ and $\bar{p}$ are continuous.

Fig. 1 shows the phase diagram at unitarity for a populationimbalanced Fermi gas in a trap. Here $1 / k_{F} a=0$, where $a$ is the $s$-wave scattering length between fermions. Phase separation (labeled PS) occupies the lower $T$ portion of the phase diagram, where the gap $\Delta$ jumps abruptly to zero at some trap

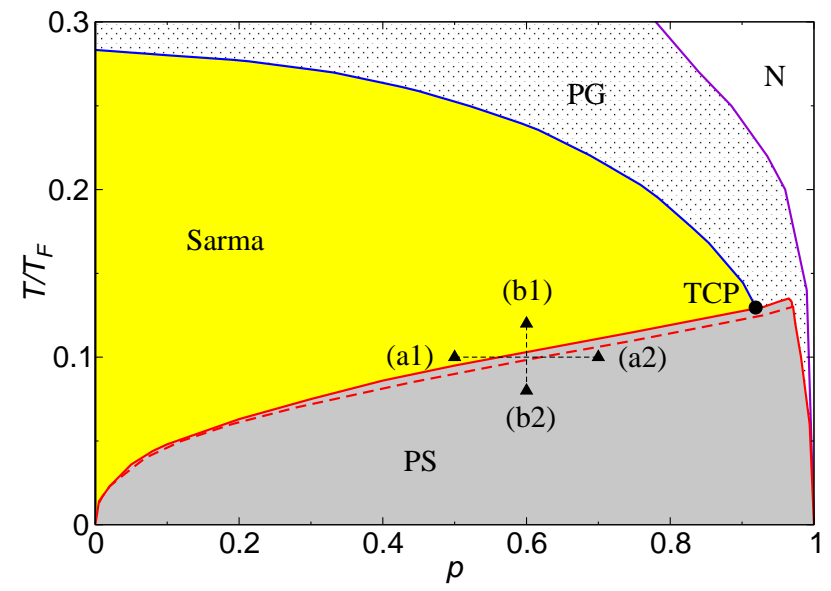

Figure 1: (Color online) Phase diagram of a population-imbalanced Fermi gas in a harmonic trap at unitarity. The solid lines separate different phases. Above the (red) dashed line but within the PS phase, the superfluid core does not touch the domain wall. Here "PG" indicates the pseudogapped normal phase. The black dot labeled "TCP" indicates the tricritical point. The four points indicated by the triangles labeled (a1)-(b2) correspond to the density profiles in Fig. 2

radius. At intermediate $T$, there is a (yellow-shaded) Sarma phase, where $\Delta$ vanishes continuously within the trap. It evolves into a (dotted) pseudogap (PG) phase as the superfluid core vanishes at higher $T$. A normal $(\mathrm{N})$ phase without pairing always exists at even higher $T$. In contrast with earlier work [11, 12], here we include pair fluctuations or non-condensed pairs, which are essential in order to obtain quantitatively correct values for $T_{c}$ as well as for the finite temperature particle density profiles [6, 13].

We stress that with or without the trap, the PS phase is the ground state at unitarity for any polarization. The differences between the trap and homogeneous phase diagrams are principally quantitative. The finite temperature boundary between the Sarma and PS phase is shifted as a result of the trap potential, allowing the Sarma state to exist at low $T$ and low polarizations. The location of the tricritical point (TCP) also shifts from low polarization in the uniform case (not shown) to high polarization in the trapped case. Away from $p \equiv 0$ there is a minimum temperature required to arrive at this Sarma phase, as a consequence of the well documented negative $T=0$ superfluid density.

In Fig. 2 we present four representative density profiles which show the behavior on different sides of the transition line between the PS and Sarma phases. The (a1)-(a2) pair have the same $T$ but different $p$, while the (b1)-(b2) pair have the same $p$ but different $T$. We have chosen these points some distance from the transition line in order to illustrate the differences. It should be noted, however, as points (a1) and (b1) (which are within the Sarma phase), move closer to the transition line, $\Delta$ drops rapidly (but not discontinuously), as a precursor to abrupt phase separation. The transition from the pure Sarma phase to the PS phase is, thus, a relatively smooth one. These LDA-based calculations should apply to situations where the trap geometry is reasonably isotropic, as for exam- 


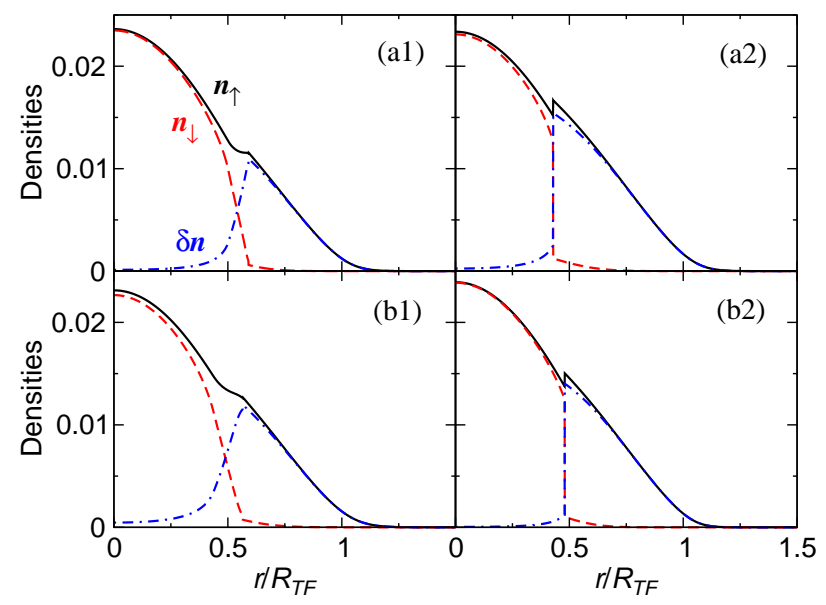

Figure 2: (Color online) Three-dimensional density profiles corresponding to the four points labeled in the phase diagram at unitarity shown in Fig. 1 (a1) $T=0.1 T_{F}, p=0.5$. (a2) $T=0.1 T_{F}$, $p=0.7$. (b1) $T=0.12 T_{F}, p=0.6$. (b2) $T=0.08 T_{F}, p=0.6$. The (black) solid, (red) dashed, and (blue) dot-dashed lines correspond to $n_{\uparrow}, n_{\downarrow}$, and $\delta n$, respectively. Here $R_{T F}=\sqrt{2 E_{F} / m \bar{\omega}^{2}}$ is the Thomas-Fermi radius, and the units for density are $k_{F}^{-3}$.

ple in the MIT experiments [1, 3] and where one might be able to ignore surface energy contributions. On the other hand, this behavior appears at odds with recent experiments from Rice [2, 4] which report pronounced changes in the aspect ratio of the profile as the transition line is crossed. These changes have been attributed to extreme trap anisotropy and associated interface energy effects [21].

In a different class of experiments it was proposed that by measuring densities at the center of the trap as one sweeps $T$ or $p$, one can infer when the Sarma-PS transition line is crossed [22] as well as where superfluid transition [i.e., $T_{c}(p)$ ] occurs [4]. As in these experiments, we plot the ratio $n_{\uparrow} / n_{\downarrow}$ at the trap center as a function of $T$ and $p$ in Fig. 3. As shown in Fig. 3 a, when $p$ is fixed at a relatively high value while sweeping $T$, the curve starts at 1 in the PS regime at low $T$, and begins to increase when the the transition line to the Sarma phase is crossed. This behavior reflects the fact that Sarma states can accommodate higher core polarizations than their PS counterparts. In Fig. 3b, when instead $T$ is fixed below TCP while sweeping $p$, the curve is a straight line independent of $p$, as observed experimentally [4]. Here one can infer, that the core has equal population in both the Sarma state at low $T$ and the PS phase at any $(T, p)$. This figure also underlines the fact that PS states exist up to very high polarizations $(p>0.99)$. Finally, when $T / T_{F}$ is higher than that of the TCP (Fig. 3c), the ratio increases rapidly after the superfluidity disappears, as appears to be observed experimentally [4]. Figure 3 r reveals that when superfluidity is present, it resides in the center of the trap, expelling the excess fermions outside the core. It should be noted that if one performs the same experiment as in Fig. 3a, but with much lower polarization (not shown), we find that the crossing of the transition line will be barely observable. as is necessary in order to be consistent with Fig. 3b. No matter how the Sarma-PS transition line is crossed, at low
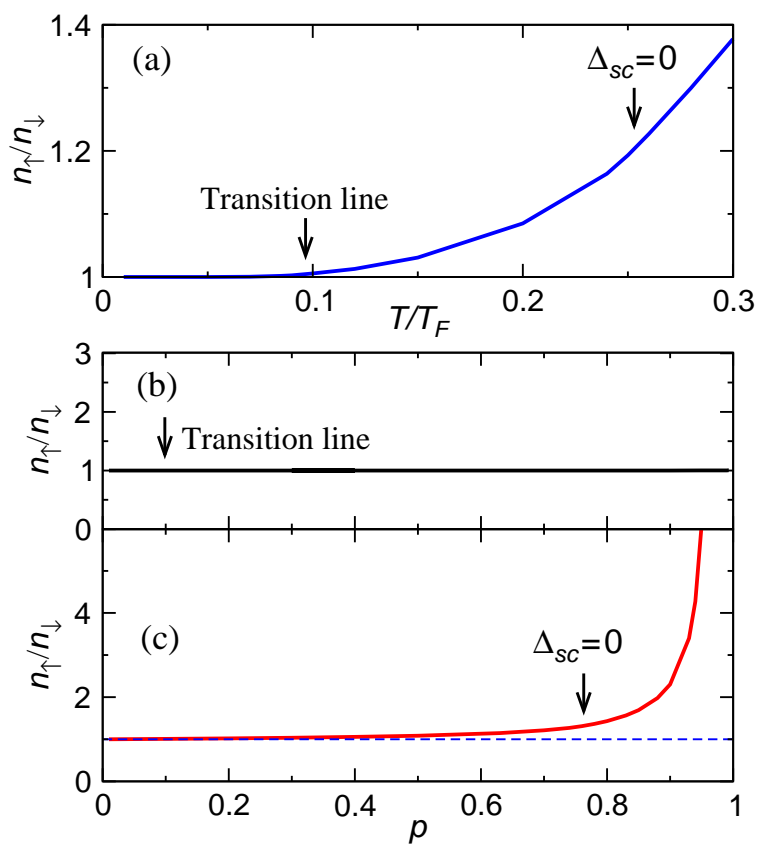

Figure 3: (Color online) Behavior of $n_{\uparrow} / n_{\downarrow}$ at the trap center at unitarity, as a function (a) of $T / T_{F}$ at fixed $p=0.5$, of $p$ at fixed (b) $T=0.05 T_{F}$ and (c) $T=0.2 T_{F}$. The (blue) dashed line in (c) indicates $n_{\uparrow} / n_{\downarrow}=1$. The arrows labeled "Transition line" indicate where the PS-Sarma transition occurs, and the arrows labeled " $\Delta_{s c}=0$ " indicate where superfluid condensate disappears in the Sarma state.

$p$ (and thus low $T$ ), the ratio $n_{\uparrow} / n_{\downarrow}$ remains 1 . Therefore, this class of experiments may not map out the transition curve for all $p$.

Our theory can be generalized to address the whole of BECBCS crossover. As one passes from unitarity towards the $\mathrm{BEC}$ regime we find the fraction of the PS phase in the phase diagram decreases progressively, disappearing at $1 / k_{F} a \approx$ 2.04. For stronger couplings, the superfluid contribution to the phase diagram consists only of the Sarma phase, at all $T$. The same observation at $T=0$ was first reported in Ref. [23]. This can also be inferred from the homogeneous phase diagram in Ref. [24], which shows that the Sarma state is stable at low $T$ for any $p$ providing $1 / k_{F} a \gtrsim 2.3$.

In Fig. 4 we present the phase diagram for a populationimbalanced trapped gas on the BCS side of resonance, where $1 / k_{F} a=-0.5$. It differs significantly from the unitary case and is, in many ways even more rich. Importantly, at $T=0$, the PS phase is no longer stable at very high $p$. We understand this by noting that in the BCS regime, the pairing interaction is relatively weak and the gap $\Delta$ small. At sufficiently high $p$, we have $h>\Delta(r=0)$ so that an unpolarized BCS superfluid core can no longer be sustained.

Equally important is the fact that in this BCS case, the Sarma and PS phases do not connect and an intermediate phase appears between the Sarma and PS states. As a consequence, the boundaries of the Sarma, PS, and (pseudogapped or unpaired) normal phases do not meet except possibly at $p=T=0$. We presume that this intermediate phase is a 


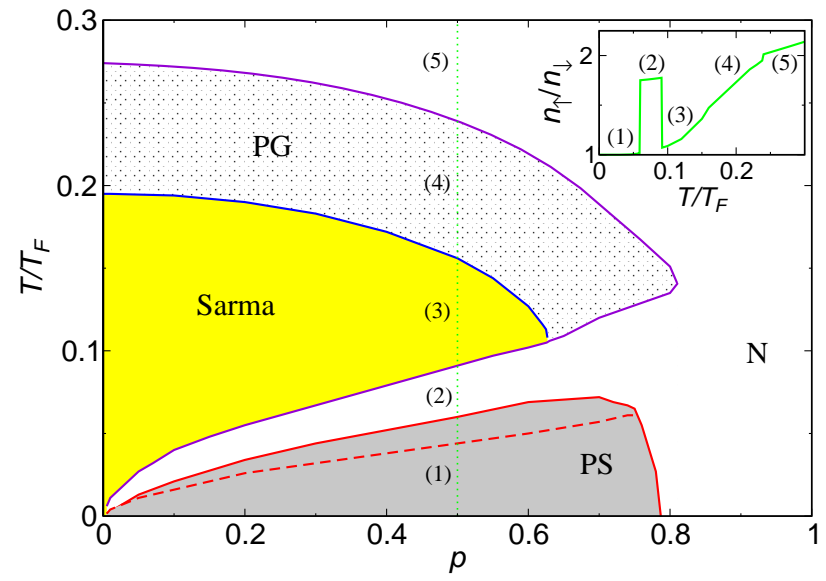

Figure 4: (Color online) Phase diagram of a polarized Fermi gas, as in Fig. 1 except that $1 / k_{F} a=-0.5$. Here the Sarma and PS phases are separated by an intermediate normal regime. The (green) dotted line indicates a sweep of $T$ at $p=0.5$, with five possible structures labeled: (1) PS, (2) N, (3) Sarma, (4) PG, (5) N. Shown in the inset is $n_{\uparrow} / n_{\downarrow}$ at the trap center as a function of $T / T_{F}$.

normal Fermi gas $(\mathrm{N})$. To understand its appearance, we note that as the BCS regime is approached, (i) phase separation becomes problematic because finite temperature (which enters via $T / \Delta$ ) has a stronger effect, allowing the polarization to penetrate into the center of the core and thereby making PS more difficult. (ii) In addition, the intermediate temperature Sarma phase becomes more fragile as the pairing is weakened.
As a result the Sarma and PS states retreat from each other as seen in Fig. 4. We cannot rule out LOFF-like states as an alternative candidate in place of N. However, we have found [19] these phases in general have very low $T_{c}$ and should not persist at these higher temperatures. In the inset of Fig. 4 we plot $n_{\uparrow} / n_{\downarrow}$ at the trap center as a function of $T / T_{F}$. The $\mathrm{N}$ state between the PS and Sarma phases would be manifested by sudden jumps at the PS-N and N-Sarma boundaries. This prediction can be used to test the existence of the intermediate $\mathrm{N}$ state in the phase diagram.

We end with another prediction concerning how the "mixed normal" region of the trap, emphasized experimentally in Ref. [3], evolves with $T$. As noted in earlier work [25], within the Sarma phase, this mixed normal state consists of highly correlated non-condensed pairs which necessarily become less significant as $T$ decreases. However, with decreasing $T$, as seen from Figs. 1 and 4 the Sarma phase gives way to stable phase separation. Only in the sliver above the (red) dashed line will a phase separated gas contain a correlated mixed normal region (appearing between the superfluid core and the domain wall). Everywhere in the PS phase, there is a mixed normal region in the gas outside the domain wall, with no pairing correlations, as appears at strictly $T=0$ [8, 9]. Thus a change should occur from a highly correlated to an uncorrelated mixed normal region at large radii as $T$ is progressively decreased. Further experiments should help to address these predictions.

This work was supported by NSF PHY-0555325 and NSFMRSEC Grant No. DMR-0213745; we thank Randy Hulet, Martin Zwierlein and Cheng Chin for useful conversations.
[1] M. W. Zwierlein, A. Schirotzek, C. H. Schunck, and W. Ketterle, Science 311, 492 (2006).

[2] G. B. Partridge, W. Li, R. I. Kamar, Y. A. Liao, and R. G. Hulet, Science 311, 503 (2006).

[3] M. W. Zwierlein, C. H. Schunck, A. Schirotzek, and W. Ketterle, Nature (London) 442, 54 (2006).

[4] G. B. Partridge, W. Li, Y. A. Liao, R. G. Hulet, M. Haque, and H. T. C. Stoof, Phys. Rev. Lett. 97, 190407 (2006); R. G. Hulet, private communications.

[5] M. M. Forbes, E. Gubankova, W. V. Liu, and F. Wilczek, Phys. Rev. Lett. 94, 017001 (2005).

[6] Q. J. Chen, J. Stajic, S. N. Tan, and K. Levin, Phys. Rep. 412, 1 (2005); K. Levin and Q. J. Chen, e-print cond-mat/0611104.

[7] D. E. Sheehy and L. Radzihovsky, Phys. Rev. Lett. 96, 060401 (2006).

[8] T. N. De Silva and E. J. Mueller, Phys. Rev. A 73, 051602(R) (2006).

[9] M. Haque and H. T. C. Stoof, Phys. Rev. A 74, 011602(R) (2006).

[10] Q. J. Chen, Y. He, C.-C. Chien, and K. Levin, e-print cond-mat/0608662

[11] K. Machida, T. Mizushima, and M. Ichioka, Phys. Rev. Lett. 97, 120407 (2006).

[12] K. B. Gubbels, M. W. J. Romans, and H. T. C. Stoof, Phys. Rev. Lett. 97, 210402 (2006).
[13] J. Stajic, Q. J. Chen, and K. Levin, Phys. Rev. Lett. 94, 060401 (2005).

[14] M. M. Parish, F. M. Marchetti, A. Lamacraft, and B. D. Simons (2006), cond-mat/0605744.

[15] P. Nozières and S. Schmitt-Rink, J. Low Temp. Phys. 59, 195 (1985).

[16] T. N. De Silva and E. J. Mueller, Phys. Rev. A 73, 051602(R) (2006).

[17] P. Fulde and R. A. Ferrell, Phys. Rev. 135, A550 (1964); A. I. Larkin and Y. N. Ovchinnikov, Zh. Eksp. Teor. Fiz. 47, 1136 (1964) [Sov. Phys. JETP 20, 762 (1965)].

[18] H. Caldas, Phys. Rev. A 69, 063602 (2004).

[19] Y. He, C.-C. Chien, Q. J. Chen, and K. Levin, e-print cond-mat/0610274

[20] F. Chevy, Phys. Rev. Lett. 96, 130401 (2006).

[21] T. N. De Silva and E. J. Mueller, Phys. Rev. Lett. 97, 070402 (2006).

[22] Y. Shin, M. W. Zwierlein, C. H. Schunck, A. Schirotzek, and W. Ketterle, Phys. Rev. Lett. 97, 030401 (2006).

[23] P. Pieri and G. C. Strinati, Phys. Rev. Lett. 96, 150404 (2006).

[24] C.-C. Chien, Q. J. Chen, Y. He, and K. Levin, Phys. Rev. Lett. 97, 090402 (2006).

[25] C.-C. Chien, Q. J. Chen, Y. He, and K. Levin, Phys. Rev. A 74, 021602(R) (2006). 